\title{
Microscopic Findings Location
}

National Cancer Institute

\section{Source}

National Cancer Institute. Microscopic Findings Location. NCI Thesaurus. Code C117593.

The site in or on the body at which the microscopic assessment test or result is performed or collected. 